\title{
Eficiencia del lagunaje profundo como tratamiento para la eliminación de metales pesados en aguas residuales de tipo urbano-industrial
}

\author{
M. Arauzo ${ }^{1}$ M. Valladolid ${ }^{2}$, O. Cedenilla ${ }^{1} \&$ C. Noreña ${ }^{2}$ \\ ${ }^{1}$ Centro de Ciencias Medioambientales C.S.I.C., Serrano 115 dpdo., 28006 Madrid, España \\ Fax: 34915640800 Teléfono: 3491 7452500. E-mail: mercedes@ccma.csic.es \\ ${ }^{2}$ Museo Nacional de Ciencias Naturales C.S.I.C., José Gutiérrez Abascal 2, 28006 Madrid, España. \\ Fax: 34915645078 Teléfono: 34914111328
}

\section{RESUMEN}

En 1996 se puso en marcha una laguna experimental para la regeneración de aguas residuales, de $4.75 \mathrm{~m}$ de profundidad, alimentada en continuo con agua residual de origen urbano-industrial previamente tratada. Se han realizado diversos estudios a fin de conocer su dinámica interna y de optimizar su rendimiento. El lagunaje profundo, situado en serie con el efluente de una depuradora (tratamiento físico-químico + fangos activos) es una tecnología de tipo terciario que, mediante un procedimiento natural y sin gasto energético adicional, mejora notablemente la calidad química y sanitaria del agua regenerada, tanto para una posible reutilización agrícola, como para su incorporación a un cauce fluvial. La laguna profunda actúa, además, como una trampa de sedimento para los metales pesados. Los procesos de asociación de estos elementos traza con la materia particulada por adsorción, precipitación, coprecipitación o asimilación por la biota, favorecen su eliminación de la columna de agua y su acumulación en el sedimento. La dinámica natural de la laguna posibilita tales procesos. El pH, ligeramente alcalino, las condiciones reductoras, la temperatura, la materia orgánica abundante y la disponibilidad de iones inorgánicos, son las principales variables implicadas. Por otra parte, la profundidad y la amplitud del tiempo de residencia hidráulica favorecen los procesos de sedimentación. Los rendimientos medios del lagunaje profundo en la eliminación de cromo, hierro y zinc se estimaron en torno al $60 \%$.

Palabras clave: lagunaje profundo, metales pesados, aguas residuales urbano-industriales.

\begin{abstract}
An experimental treatment pond for residual waters reclamation with a depth of $4.75 \mathrm{~m}$ was set up in 1996. It was continuously fed with urban and industrial wastewater previously treated. Several studies have been carried out in order to provide a better understanding of the internal dynamics of the pond and to optimise its performance. A deep pond treatment, arranged in series with the effluent from a treatment plant (physic-chemical treatment + activated sludge) is a terciary technology which, through natural procedures and without additional energy consumption, improves substantially the chemical and sanitary quality of the water so reclaimed, making it suitable both for potential agricultural reuse as well as discharge into watercourses. In addition, a deep pond acts as a sediment trap for heavy metals. Trace elements are known to bind to particulate matter through adsorption, precipitation, coprecipitation and uptake by biota, thus favouring its removal from the water column and accumulation in the sediments. The natural dynamics of the pond makes possible such processes. $p H$, slightly alkaline, reducing conditions, temperature, abundant organic matter and availability of inorganic ions are the main variables involved. On the other hand, the depth of the pond and the length of residence time favour the sedimentation processes as well. Mean removal efficiencies for $\mathrm{Cr}$, Fe and Zn concentrations were found to be around $60 \%$.
\end{abstract}

Keywords: deep treatment pond, heavy metals, urban and industrial wastewater. 


\section{INTRODUCCIÓN}

El origen de la mayor parte de las sustancias químicas tóxicas de las aguas residuales está ligado a actividades industriales conectadas a las redes de saneamiento (WHO, 1984). Desafortunadamente, la mayor parte de los sistemas colectores de aguas residuales urbanas no posibilitan la separación de los efluentes provenientes de las industrias, de modo que la situación real es que los efluentes de las zonas urbanas con polígonos industriales son generalmente de tipo mixto. Por otra parte, los sistemas convencionales de depuración de aguas residuales (tratamiento primario + secundario) presentan unos rendimientos medios para la eliminación de elementos traza que distan de poder ser considerados eficientes (U.S. EPA, 1992). Si bien es cierto que se han desarrollado procesos tecnológicos específicos para el tratamiento químico y la remoción de metales contaminantes de las aguas residuales (Palmer et al., 1988), sus aplicaciones son muy costosas y están poco generalizados, dado que requieren importantes inversiones en equipamiento, reactivos químicos, control de procesos y gastos energéticos. Sin embargo, los fundamentos químicos en los que se basan algunos de estos procesos, y más concretamente aquellos en los que el objetivo final es la precipitación, la adsorción o la reducción, también pueden darse de forma espontánea en ciertos ecosistemas acuáticos bajo determinadas condiciones ambientales naturales.

En el presente estudio se valora la eficiencia del lagunaje profundo como un sistema de tratamiento avanzado, ecológico, de bajo coste económico y de escaso mantenimiento, cuya dinámica natural proporciona las condiciones necesarias para la reducción en concentración de los metales pesados de las aguas residuales de tipo urbanoindustrial y su acumulación en el sedimento.

$\mathrm{Al}$ igual que el lagunaje convencional (depuración blanda, lagunas someras), el funcionamiento de las lagunas profundas está basado en el mismo principio de autodepuración del agua que opera en ríos y lagos. El lagunaje profundo ofrece, además, ciertas expectativas ventajosas en la regeneración de las aguas residuales urbanas respecto al lagunaje convencional: las lagunas profundas ocupan menor superficie (menor inversión en terrenos), en climas áridos o semiáridos se reducen las pérdidas por evaporación (efluente menos salinos, mejorándose la calidad para su reutilización en riego; Moreno, 1984) y, durante los meses fríos, se favorece la retención de calor (Moreno, 1991). A pesar de ello, mientras que el uso del lagunaje convencional está muy extendido y ha sido ampliamente estudiado (Instituto de Ingeniería Sanitaria, 1971; Parker, 1979; Parker et al., 1979; Uhlmann, 1979; Dinges, 1982; Middlebrooks et al., 1982; Buhr et al., 1983; Vander Borght et al. 1987; Moreno 1991; Ulloa, 1993), la utilización del lagunaje profundo es poco frecuente y sus estudios científicos escasos (Moreno, 1984; Moreno et al. 1984ab; Inca et al. 1987; Moreno et al. 1988; Soler et al., 1988; Arauzo, 2000; Arauzo, 2002; Arauzo et al., 2000; Arauzo et al., 2001).

La toxicidad potencial y la biodisponibilidad de un metal pesado depende de su propia naturaleza y de su disponibilidad en el ambiente (Wood, 1974), así como de su estado molecular (Stumm \& Morgan, 1981). El pH puede afectar a la especiación química y a la movilidad de muchos metales (Wood, 1989) y puede influir fuertemente en la adsorción o desorción por las sustancias orgánicas. El potencial de óxidoreducción también influye sobre la especiación metálica (Wood, 1989). Por ejemplo, en condiciones reductoras se produce la transformación microbiana de sulfato a sulfito, lo que provoca la precipitación de los metales pesados (Babich \& Stotzky, 1983). Respecto a los iones inorgánicos, tanto aniones como cationes pueden influir sobre la disponibilidad de los metales, como es el caso de precipitación de carbonatos insolubles o la adsorción sobre carbonato cálcico (Prosi, 1981). La temperatura influye sobre la solubilidad de los metales. La materia orgánica, tanto soluble como particulada, produce una disminución de los metales disueltos y un aumento de la concentración de metales en forma coloidal, en suspensión y en los sedimentos (Rovira, 1993). Respecto a factores de tipo 
biótico, la actividad metabólica de los microorganismos también puede ejercer un papel significativo en la movilidad de los elementos tóxicos en el ambiente (Wood, 1974).

Cuando un metal pesado llega a un sistema acuático, parte se asociará con ligandos disueltos de carácter tanto orgánico como inorgánico, mientras que otra fracción se asociará con materia particulada por adsorción, precipitación, coprecipitación o asimilación por la biota. El flujo de los metales hacia los sedimentos puede ser gravitatorio, cuando están asociados a materia particulada, o por difusión, cuando los metales disueltos en la columna de agua están en mayor concentración que en el agua intersticial del sedimento, especialmente en lagos de carácter ácido (Tessier y Campbell, 1987). La compartimentación de los metales pesados en los sedimentos depende de muchas variables, como el tipo y concentración de ligandos en el agua, la naturaleza del sustrato sólido, el potencial redox, el pH, etc. (Tessier y Campbell, 1987). Los metales se pueden asociar a diferentes fases del sedimento mediante adsorción sobre la superficie de partículas (arcillas, ácidos húmicos, óxidos hidratados de metales), unidos a carbonatos, ocluidos en óxidos hidratados de hierro o manganeso y unidos a materia orgánica (tanto viva como detrítica), a sulfuros o a la propia matriz del sedimento (Tessier y Campbell, 1987). También se encuentran en forma disuelta o coloidal en el agua intersticial. Sin embargo, el sedimento no constituye un compartimiento estanco para los metales, que pueden solubilizarse al variar alguna condición del sistema.

En los sistemas de lagunaje profundo, la dinámica biológica tiene una marcada influencia sobre el $\mathrm{pH}$ del agua, las condiciones reductoras, la materia orgánica (detrítica o en organismos vivos) y los iones inorgánicos (Arauzo, 2000; Arauzo et al., 2000), variables que pueden influir sobre el comportamiento y la biodisponibilidad de los metales pesados y de otros elementos traza potencialmente tóxicos. La laguna profunda actuaría como una gran trampa de sedimento en la que los procesos de asociación con la materia particulada (adsorción, precipitación, coprecipitación o asimilación por la biota) favorecerían la retirada de los metales de la columna de agua y su acumulación en el sedimento. La dominancia de los procesos de sedimentación en la laguna, debido a la profundidad, y los tiempos de residencia hidráulicos relativamente largos, son factores que contribuirían a potenciar la eliminación de estos elementos contaminantes.

Existen algunos estudios preliminares sobre la reducción en concentración de metales traza durante su almacenaje en embalses o lagunas de estabilización, desarrollados por Smillie y Loutit (1982) en Nueva Zelanda, Carre et al. (1986) en Francia, Baozhen (1987) en China y Kaplan et al. (1987) en Israel. Más recientemente, Juanicó et al. (1999) presentaron resultados sobre la remoción de metales pesados en dos embalses de estabilización situados en serie, con entrada del agua residual en continuo, previamente tratada mediante fangos activos (sistema que podría ser comparable al que se presenta en este trabajo).

\section{MATERIAL Y MÉTODOS}

La laguna experimental tiene $4.75 \mathrm{~m}$ de profundidad y una capacidad útil de $2 \times 200 \mathrm{~m}^{3}$. Se alimenta, en continuo, con agua residual de tipo urbanoindustrial proveniente de Arganda del Rey (Madrid), previamente tratada con físico-químico y fangos activos en la estación depuradora de dicha localidad. La laguna está ubicada en la Finca Experimental La Poveda, que gestiona el Centro de Ciencias Medioambientales (CSIC). Su planta tiene forma de pirámide truncada invertida de base cuadrada, con 10x10m en la zona más profunda y $31 \times 31 \mathrm{~m}$ en superficie. Para evitar filtraciones, su fondo y laterales están recubiertos de fieltro geotextil, sobre el que se ha dispuesto otra capa de polietileno de alta densidad. El influente está situado a $4.5 \mathrm{~m}$ de profundidad. El efluente está a $1.5 \mathrm{~m}$ de la superficie y se ubica en el lateral opuesto al influente. Los vientos dominantes son perpendiculares a la línea influente-efluente. El caudal se registra, en continuo, mediante un caudalímetro en línea de presión. 
El estudio se realizó a lo largo de un ciclo anual completo, desde septiembre de 2000 hasta octubre de 2001, y comprendió un periodo de mezcla y otro de estratificación térmica de la laguna. Se trabajó con un caudal de entrada, en continuo, de $6 \mathrm{~m}^{3} \mathrm{~h}^{-1}$, que corresponde a un tiempo teórico de residencia hidráulica de 15 días.

Con frecuencia mensual, se recogieron muestras de agua compuestas en el influente y en el efluente de la laguna. Los muestreos se realizaron siempre entre las 10 y las 11:30 de la mañana. Cada muestra compuesta procedía de la mezcla de alícuotas tomadas cada hora, durante las 24 horas previas al muestreo, mediante el empleo de muestreadores automáticos. Sobre cada una de ellas se analizaron las concentraciones totales de los siguientes elementos traza: $\mathrm{Cr}$, $\mathrm{Cu}, \mathrm{Ni}, \mathrm{Zn}, \mathrm{Al}, \mathrm{Co}, \mathrm{Cd}, \mathrm{Fe}, \mathrm{Pb}, \mathrm{Mn}, \mathrm{Ti}, \mathrm{V}, \mathrm{Mo}$, $\mathrm{Ag}, \mathrm{Li}, \mathrm{F}, \mathrm{B}, \mathrm{Se}$ y As. Las muestras para la analítica de elementos traza fueron previamente digeridas mediante ataque nítrico-perclórico, a fin de terminar las concentraciones totales de cada elemento. Asimismo, se analizaron otras variables indicadoras del rendimiento del sistema, como los sólidos en suspensión, la demanda química de oxígeno y los coliformes fecales. Con la misma frecuencia, se realizaron perfiles (metro a metro) en el centro de la laguna, de temperatura, oxígeno disuelto, $\mathrm{pH}$, conductividad y clorofila $a$. La analítica del agua se llevó a cabo según APHA (1998). Para la analítica de elementos traza se utilizó la espectrometría de emisión ICP-AES (plasma de acoplamiento inductivo) y la cromatografía iónica (IC). Los perfiles de temperatura y oxígeno disuelto se efectuaron con un oxímetro de campo YSI-57. La medida del $\mathrm{pH}$ con un pHmetro de campo HANNA HI 9023 y la de la conductividad con un conductímetro de campo HANNA HI 9033. La clorofila $a$ se analizó según el método de Marker et al. (1980), extrayéndose para ello muestras de agua del perfil de la laguna (metro a metro) mediante una bomba peristáltica sumergible. El fitoplancton se determinó mediante microscopía óptica invertida, según el método de Utermöhl (1958).

Para conocer la concentración de cromo hexavalente se realizaron análisis con frecuencia bimestral mediante el método colorimétrico del difenil-carbacida en solución ácida (APHA, 1998).

Los rendimientos en la reducción en concentración de elementos traza, de sólidos en suspensión, de coliformes fecales y de demanda química de oxígeno (expresados en tanto por ciento) se calcularon mediante la expresión: "(concentración en el influente - concentración en el efluente) $x 100$ / concentración en el influente".

A fin de conocer los cambios en la dinámica de la laguna durante la noche, se realizaron muestreos nocturnos adicionales a escala horaria en los momentos más representativos de la mezcla y de la estratificación (Arauzo, 2002).

Con el objetivo de evaluar la tasa de acumulación de elementos traza en el sedimento de la laguna, se realizó un ensayo de campo que consistió en la instalación un total de 16 trampas experimentales de sedimento en el fondo de la misma. Cada trampa, de forma tubular, con 4.3 $\mathrm{cm}$ de diámetro interno, $50 \mathrm{~cm}$ de altura interna y $726 \mathrm{~cm}^{3}$ de capacidad útil, se construyó en PVC y fue lastrada en el fondo. Las 16 trampas se colocaron, desprovistas de tapón, previamente rellenas de agua destilada y debidamente lastradas, en la zona central de la laguna, a $4.75 \mathrm{~m}$ de profundidad. El ensayo se inició el 5 de octubre de 2000 y las trampas fueron siendo extraídas, en lotes de tres (tres réplicas en cada muestreo), con frecuencia bimestral. Sobre los contenidos de cada una de ellas se determinó el residuo seco total a $103-105^{\circ} \mathrm{C}$ y la masa de cada elemento traza por $\mathrm{Kg}$ de materia seca de sedimento $(\mathrm{Cr}, \mathrm{Cu}, \mathrm{Ni}, \mathrm{Zn}, \mathrm{Al}, \mathrm{Co}, \mathrm{Cd}, \mathrm{Fe}, \mathrm{Pb}$, $\mathrm{Hg}, \mathrm{Mn}, \mathrm{Ti}, \mathrm{V}, \mathrm{Mo}, \mathrm{Ag}, \mathrm{Li}, \mathrm{F}, \mathrm{B}, \mathrm{Se}, \mathrm{As})$. La analítica se realizó mediante espectrometría de emisión ICP-AES. Cada muestra de sedimento fue previamente digerida, mediante ataque nítricoperclórico, a fin de determinar las concentraciones totales de cada elemento.

En lo referente a la reutilización de efluentes de agua residual regenerada para riego agrícola, la Organización Mundial de la Salud (WHO, 1984) recomienda, como estándar, el cumplimiento de las concentraciones máximas permitidas de elementos traza para el agua de riego propuestas por la National Academy of Science 
\& National Academy of Engineering (1973), referencia que será utilizada en la valoración de los resultados de este trabajo de investigación hasta que se produzca un desarrollo de las normativas europea, nacional y de las Comunidades Autónomas en este sentido. Organizaciones como la FAO (Ayers \& Westcot, 1985) y la U.S.EPA (1992) han adoptado y recomiendan estas mismas directrices.

\section{RESULTADOS}

En la figura 1 se representan los diagramas de isolíneas para la temperatura del agua, el oxígeno disuelto, el $\mathrm{pH}$, la clorofila $a$ y la conductividad en la laguna experimental. Durante los meses más cálidos y debido a la profundidad, se desarrolló una estratificación térmica estable, que se inició en abril, se estabilizó en junio (hacia $1.5 \mathrm{~m}$ de
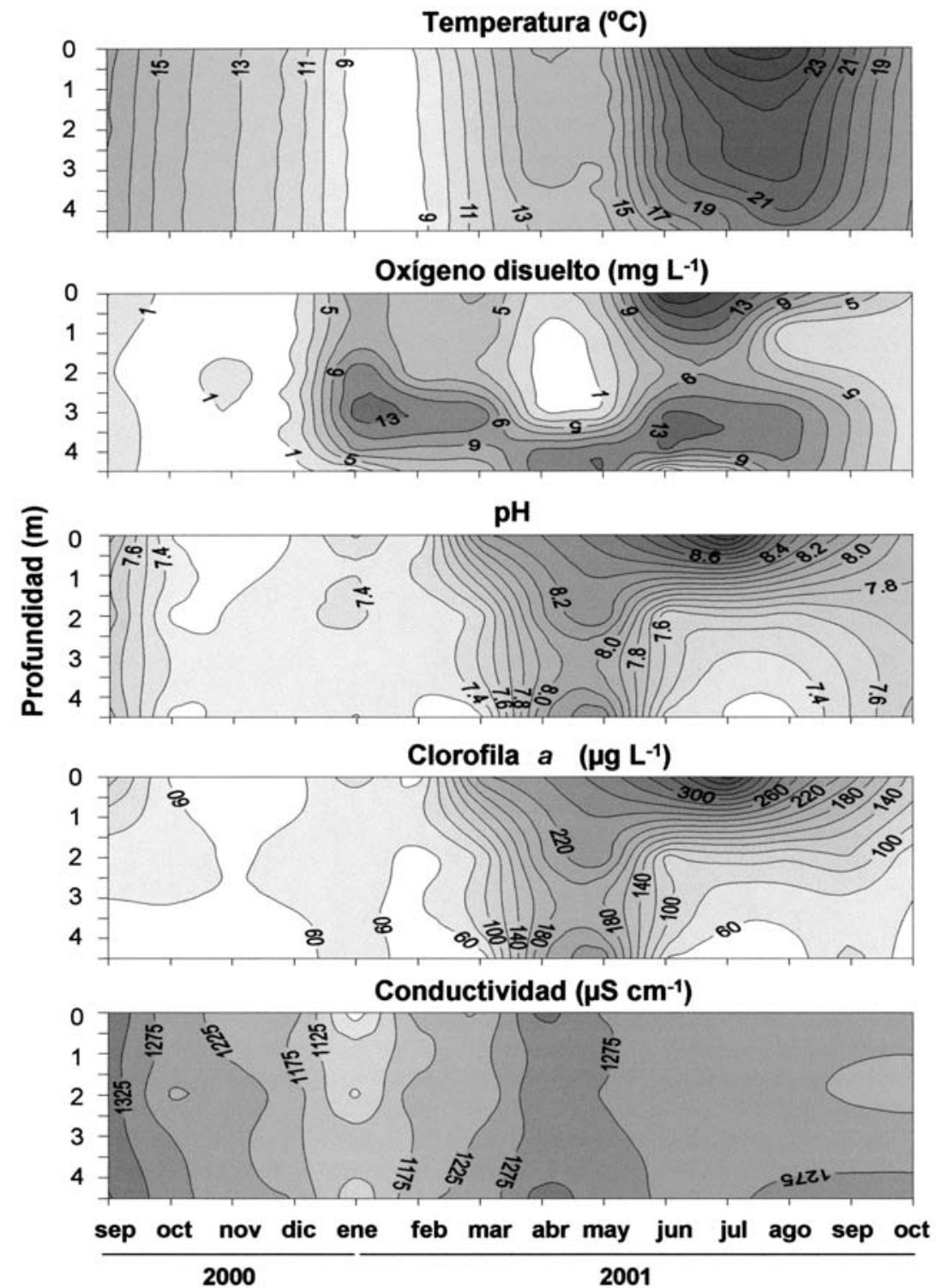

Figura 1: Diagramas de isolíneas para la temperatura del agua, el oxígeno disuelto, el pH, la clorofila $a$ y la conductividad en la laguna profunda desde septiembre de 2000 hasta octubre de 2001. Contour plots of water temperature, dissolved oxygen, pH, Chlorophyll a and conductivity in the pond from September 2000 to October 2001. 
profundidad) y finalizó al terminar septiembre. Durante el resto del ciclo anual la laguna permaneció mezclada en toda su profundidad. La clorofila $a$ y el pH alcanzaron valores elevados en toda la columna de agua durante el periodo en el que la termoclina no se hallaba completamente estabilizada y, en el epilimnion, durante el resto de la estratificación. Los grupos Euglenophyceae y Cryptophyceae fueron los de biomasa más abundante durante la mezcla, mientras que el grupo
Chlorophyceae dominó durante la estratificación (representado por los órdenes Chlorococcales y Volvocales). Las concentraciones más bajas de oxígeno disuelto se dieron durante el periodo más frío de la mezcla. Las más elevadas, en el epilimnion, durante la estratificación, en concordancia con la distribución de productores primarios. Asimismo, entre 3 y $4.5 \mathrm{~m}$ y coincidiendo con el estrato de máxima abundancia del alga Cryptomonas erosa Ehrenberg, se observaron
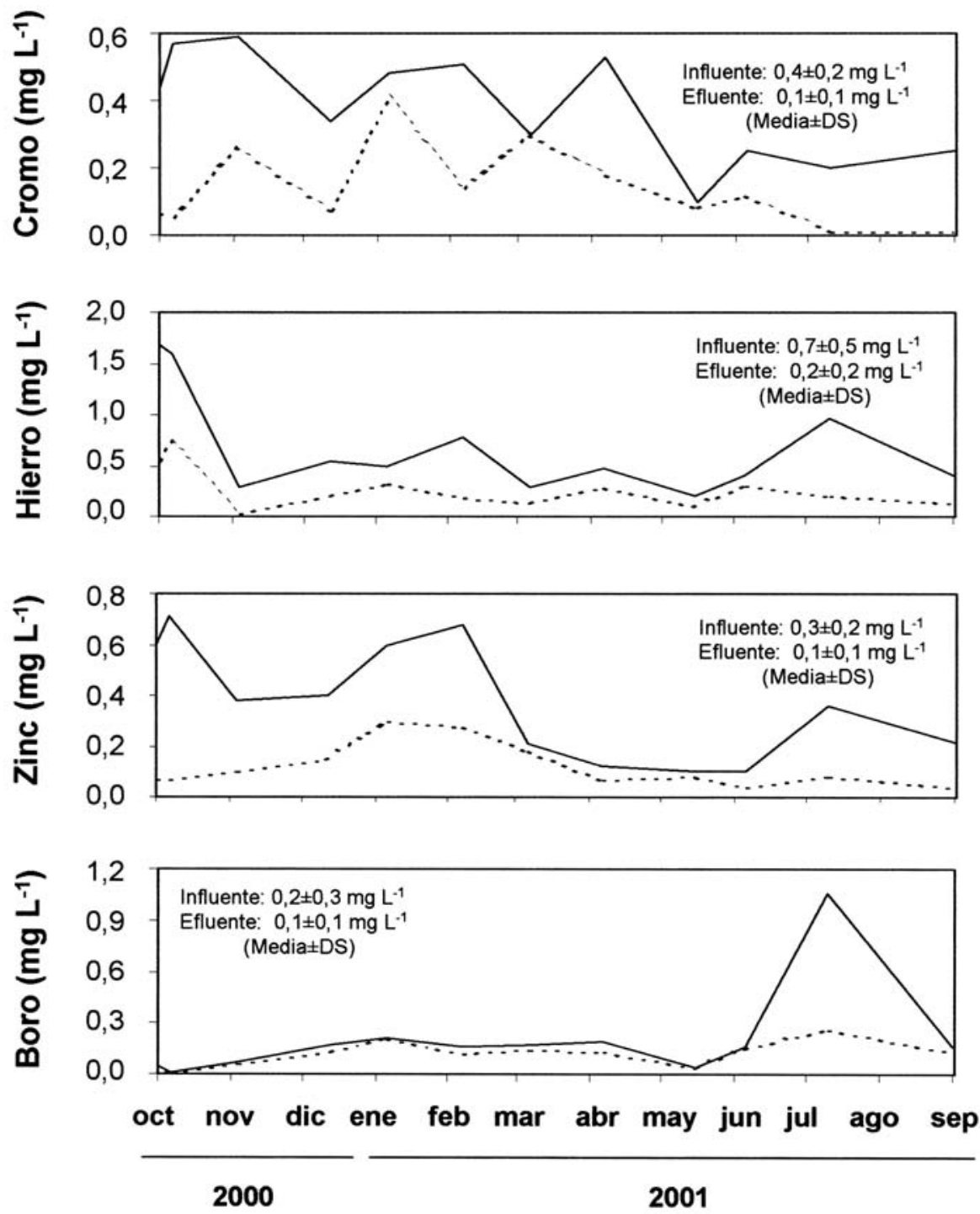

Figura 2: Curso temporal de los elementos traza detectados en el influente (línea continua) y el efluente (línea discontinua) de la laguna. Time course of trace elements detected in the influent (solid line) and the effluent (dashed line) of the pond. 
valores elevados de oxígeno desde febrero a junio. Sin embargo, debe considerarse que las concentraciones de oxígeno disuelto representadas en la figura 1 corresponden a muestreos diurnos, pudiendo cambiar las condiciones durante las horas de la noche. Muestreos nocturnos adicionales realizados a escala horaria (Arauzo, 2002) revelaron la existencia de condiciones anaerobias durante la noche, a partir de $2 \mathrm{~m}$ de profundidad (incluso durante los "blooms" de fitoplancton, con sobresaturación de oxígeno durante el día). La conductividad no mostró grandes fluctuacio- nes, aunque se observaron valores ligeramente inferiores durante los meses más fríos.

En la figura 2 se representan las concentraciones de cromo, hierro, zinc y boro en el influente y en el efluente de la laguna, únicos elementos traza detectados en sus aguas durante el periodo de estudio. Se aprecia una clara reducción en sus concentraciones medias anuales en el efluente, además de un efecto de disminución sobre los estadísticos de dispersión. En la tabla 1 se muestran las concentraciones medias en el influente y los rendimientos medios de la laguna en la reduc-

Tabla 1: Concentraciones en el influente y rendimientos ((Influente-Efluente)100/Influente) de la laguna en la reducción de demanda química de oxígeno, sólidos en suspensión, coliformes fecales, cromo, hierro, zinc y boro durante la mezcla (septiembre de 2000 hasta marzo de 2001), la estabilización de la termoclina (abril y mayo de 2001) y la estratificación (junio a septiembre de 2001). Para cada variable, se han comparado los rendimientos durante los tres periodos mediante un análisis de la varianza de 1 factor; los valores sucedidos de símbolos distintos presentaron diferencias significativas entre periodos para $\mathrm{p}<0.05$. DS: desviación estándar. Inflow concentrations and performance ((InfluentEffluent)100/Influent) of the pond for reduction of chemicaloxygen demand, suspended solids, faecal coliforms, chromium, iron, zinc and boron during mixing (from September 2000 to March 2001), thermocline stabilization (April and March 2001) and stratification (from June to September 2001). For each variable one-way analysis of variance has been performed to compare performances during the three periods, numbers followed by different symbols indicate a significant difference at the value of $p<0.05$. DS: standard deviation.

CONCENTRACIONES EN EL INFLUENTE:

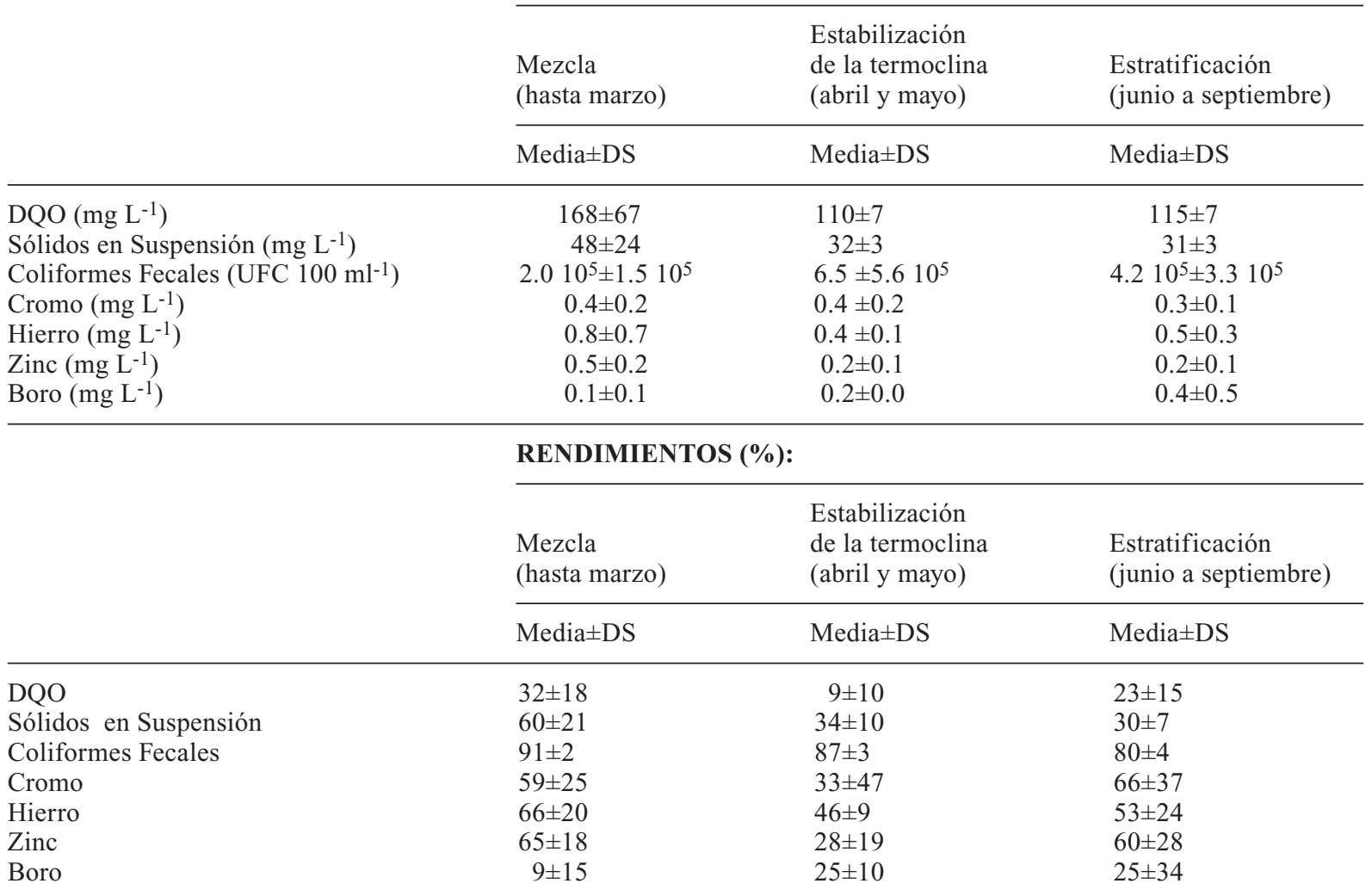



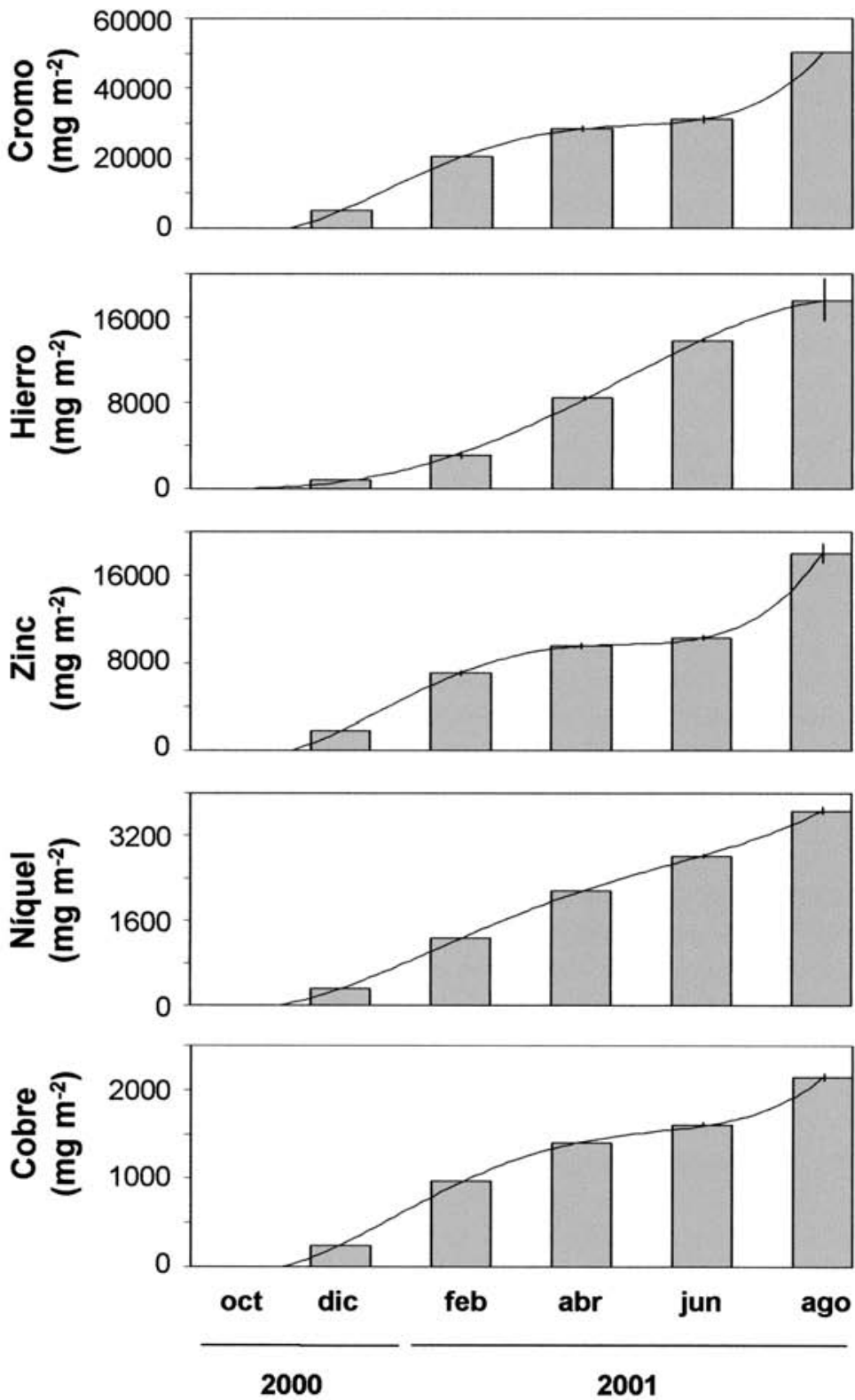

Figura 3: Elementos traza acumulados en el sedimento de la laguna durante el período de ensayo con las trampas de sedimento (primera parte). Se representan las líneas de tendencia. Barras verticales: desviación estándar. Trace elements accumulated in the sediment of the pond during the sediment traps study (first part). Trend lines have been plotted. Vertical bars: standard deviation.

ción de elementos traza y de otras variables indicadoras del funcionamiento del sistema (sólidos en suspensión, coliformes fecales, demanda química de oxígeno) durante la mezcla (desde septiembre de 2000 hasta marzo 2001), la estabilización de la termoclina (abril y mayo de 2001) y la estratificación de verano (de junio a septiembre de 2001). Mediante un análisis de la varianza de 1 factor, para cada variable, se han comparado los rendimientos durante los tres periodos, observándose mejores rendimientos en reducción de sólidos en suspensión y coliformes fecales durante la 

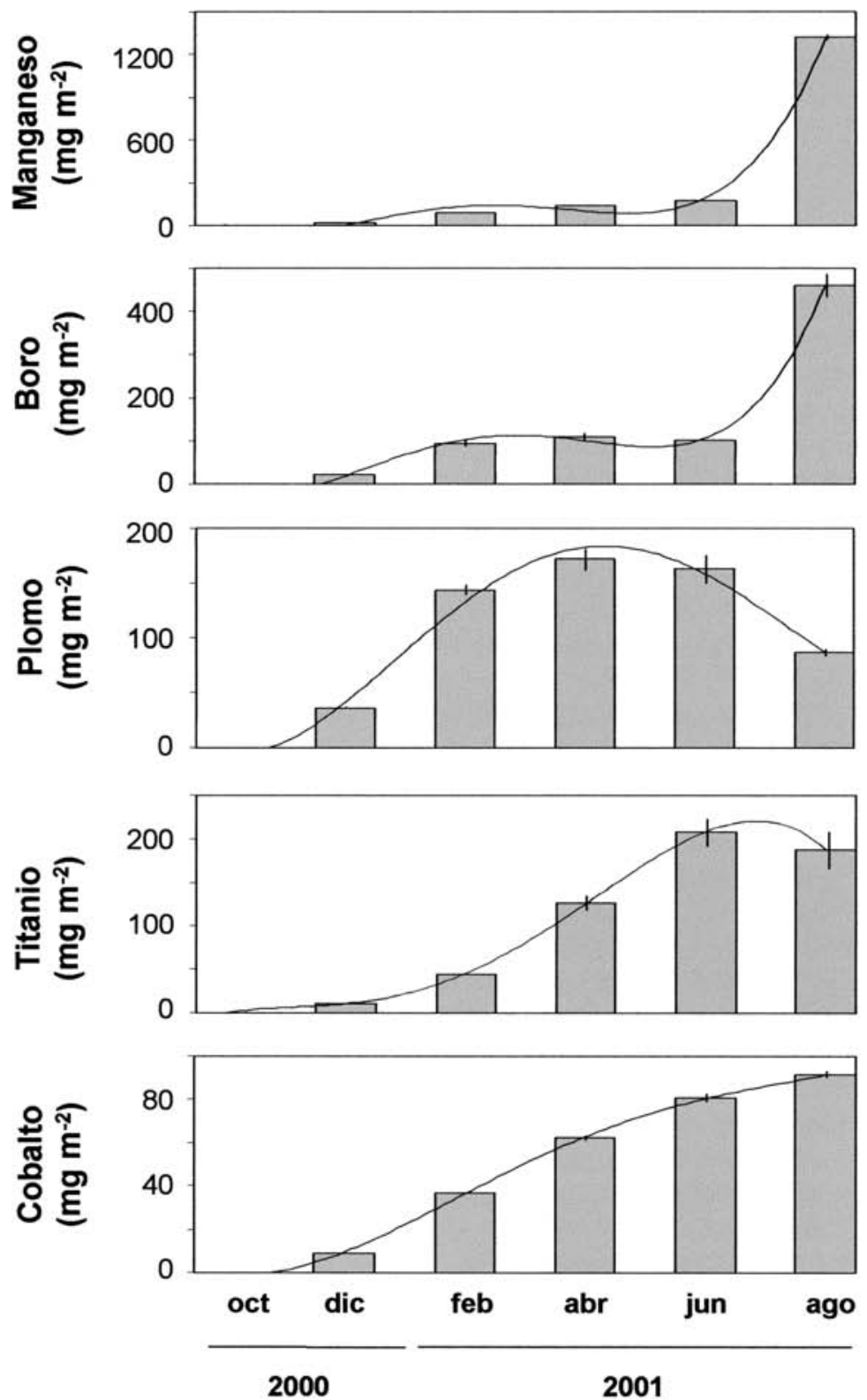

Figura 4: Elementos traza acumulados en el sedimento de la laguna durante el período de ensayo con las trampas de sedimento (segunda parte). Se representan las líneas de tendencia. Barras verticales: desviación estándar. Trace elements accumulated in the sediment of the pond during the sediment traps study (second part). Trend lines have been plotted. Vertical bars: standard deviation.

mezcla (para $\mathrm{p}<0.05$ ). No se dieron, sin embargo, diferencias significativas entre periodos para la remoción de elementos traza, salvo en el caso del zinc, con valores significativamente inferiores durante la estabilización de la termoclina.
No se observaron correlaciones significativas entre las concentraciones de elementos traza en el efluente de la laguna y el resto de las variables (concentraciones de elementos traza en el influente y temperatura, oxígeno disuelto, $\mathrm{pH}$, 
clorofila $a$, conductividad, demanda química de oxígeno y sólidos en suspensión en la laguna), salvo en los casos del cromo y el zinc en el efluente, que mostraron unas correlaciones de -0.58 y -0.65 con la temperatura, respectivamente (significativas para $\mathrm{p}<0.05$ ).

\section{Ensayo con las trampas de sedimento}

La tasa anual de acumulación de sedimento en la laguna se estimó en $5.3 \pm 0.3 \mathrm{kgMS} \mathrm{m}^{-2}$ año- $^{-1}$ (media \pm desviación estándar).

Los elementos traza que se detectaron en el sedimento de las trampas fueron los siguientes: cromo, hierro, zinc, níquel, boro, cobre, manganeso, plomo, titanio y cobalto. En las figuras 3 y 4 se representa la masa acumulada de cada elemento traza por unidad de superficie. Se observan tres tipos de curvas de tendencia: la primera, próxima a la linealidad (hierro, níquel, cobre y cobalto), la segunda, ascendente pero con periodos de acumulación variable (cromo, zinc, boro y manganeso) y la tercera, ascendente hasta junio y ligeramente descendente al final (plomo y titanio).

No se observaron correlaciones significativas entre la masa de cada elemento traza, acumulada bimestralmente por cada kilogramo de materia seca de sedimento (valor obtenido en cada muestreo de trampas, menos el valor del muestro anterior) y otras variables del sistema (temperatura, oxígeno disuelto, $\mathrm{pH}$, clorofila $a$, conductividad, demanda química de oxígeno y sólidos en suspensión en la laguna y concentraciones de elementos traza en el influente y en el efluente), salvo en el caso del boro en el sedimento de las trampas y el boro en el influente, con una correlación de 0.93 (significativa para $\mathrm{p}<0,01$ ).

El cromo fue el elemento que presentó la concentración más elevada en el sedimento, con $12.2 \pm 0.2 \mathrm{~g} \mathrm{Kg}^{-1} \mathrm{MS}$ (equivalente a $50.1 \pm$ $0.7 \mathrm{~g} \mathrm{~m}^{-2}$ ), acumulados durante un periodo de 10 meses. Al cromo lo siguieron el zinc y el hierro. En un orden de magnitud inferior, el níquel, el cobre y el manganeso y, en dos órdenes de magnitud, el boro, el plomo, el titanio y el cobalto. Considerando que la concentración media anual de cromo en el efluente de la planta depuradora (tratamiento físico-químico más fangos activos, excluyendo el tratamiento final mediante lagunaje profundo) fue de $0.4 \mathrm{mg} \mathrm{L}^{-1}$ (Fig. 2) y que dicha planta depuradora procesa aproximadamente $15000 \mathrm{~m}^{3}$ día $^{-1}$, se estimó un valor medio de $2.2 \mathrm{tm}$ de cromo total (del cual el $63 \pm 21 \%$ se emite en forma disuelta, como cromo hexavalente) que puede estarse repartiendo anualmente a lo largo de la cuenca receptora (río Jarama) y ecosistemas aledaños, cuando el tratamiento final mediante lagunaje no es aplicado.

\section{DISCUSIÓN}

La dinámica temporal de los parámetros bióticos y abióticos en la laguna fue similar a la observada durante otros ciclos anuales estudiados con anterioridad, bajo diferentes condiciones de manejo, observándose una clara respuesta a la estacionalidad y a la alternancia mezcla-estratificación (Arauzo, 2000; Arauzo et al., 2000; Arauzo et al., 2001).

El lagunaje profundo, situado en serie con el efluente de una depuradora convencional, es un sistema de tratamiento que mejora notablemente la calidad del agua regenerada, tanto para una posible reutilización agrícola, como para su incorporación a un cauce fluvial. Dado que la laguna experimental recibe aguas residuales de tipo urbano-industrial (provenientes de Arganda del Rey, Madrid, con un importante polígono industrial), esta modalidad de lagunaje propició, además, la retención en su sedimento de determinados elementos traza potencialmente tóxicos para los ecosistemas receptores (ecosistemas fluviales, terrenos de cultivo).

Cuanto más irregular en el tiempo es la entrada de un contaminante, mayor es el error en la estimación del rendimiento del sistema para su eliminación, requiriéndose un diseño de mayor precisión en el muestreo. Por este motivo, una gran parte de los elementos traza acumulados en el sedimento no fueron identificados en el agua durante los muestreos con frecuencia mensual (Fig. 2), no habiendo sido posible estimar del ren- 
dimiento de la laguna en la eliminación de los mismos. El ensayo con las trampas de sedimento permitió la detección y cuantificación de estos metales (Figs. 3 y 4 ), quedando probada la capacidad del lagunaje profundo para su retención, aun no habiendo sido detectados en agua. Según el grado de contaminación por cromo, zinc, níquel y cobre alcanzado en el sedimento de la laguna, éste puede clasificarse dentro del rango de sedimentos fuertemente contaminados (U.S. EPA, 1982, en: Hamdy \& Post, 1985). Sin embargo, el vaciado de la laguna y la gestión de sus fangos como residuo tóxico sólo serían necesarios tras largos periodos de funcionamiento (15-20 años).

Los mecanismos implicados en la remoción de elementos traza durante el lagunaje profundo forman parte de los procesos naturales que tienen lugar en este tipo de sistema, y están relacionados con el $\mathrm{pH}$ (siempre ligeramente alcalino en la laguna), con las condiciones reductoras (si no se alcanzan durante el día, se dan siempre durante la noche, a partir de $2 \mathrm{~m}$ de profundidad), con la materia orgánica abundante (detrítica o en organismos vivos) y con la presencia de iones inorgánicos (Prosi, 1981; Tessier \& Campbell, 1987; Wood, 1989; Rovira, 1993). La suficiente estabilidad estos parámetros, la dominancia de los procesos de sedimentación debido a la profundidad y la amplitud del tiempo de residencia hidráulica, son aspectos que favorecen la consecución de los procesos de sedimentación de los metales.

Durante el tratamiento de lagunaje profundo, los rendimientos medios anules para la eliminación de cromo, hierro y zinc se estimaron en torno al $60 \%$. Si a estos resultados se suman los habitualmente obtenidos durante los tratamientos previos [físico-químico (cloruro férrico), con eficiencias medias para la remoción de cromo, hierro y zinc del $87 \%$, el $43 \%$ y el $72 \%$, y fangos activos, con eficiencias medias del $55 \%$, el $65 \%$ y el $75 \%$, respectivamente (U.S.EPA, 1992)], se obtendrían unos rendimientos totales del $98 \%$ para el cromo, del $92 \%$ para el hierro y el $97 \%$ para el zinc, durante el proceso completo de regeneración (físico-químico + fangos activos + lagunaje profundo). En Kishon Reservoirs (Israel), un sistema para almacenamiento y reuti- lización de aguas residuales formado por dos embalses de $10.4 \mathrm{~m}$ de profundidad máxima, situados en serie y abastecidos en continuo con agua pretratada mediante fangos activos, Juanicó et al. (1999) obtuvieron reducciones del $75 \%$ para el cromo y del $65 \%$ para el zinc.

El cromo (proveniente de industrias de curtiduría y recubrimientos metálicos) fue el contaminante más abundante en las aguas residuales estudiadas, además del más preocupante desde el punto de vista medioambiental, puesto que está clasificado como de carácter muy tóxico y relativamente disponible (Wood, 1974), Su forma más peligrosa es la hexavalente, cuyos efectos cancerígenos sobre la salud humana han sido ampliamente descritos (U.S. EPA, 1998). En este trabajo se aportan resultados que avalan el interés del lagunaje profundo como una alternativa barata y ecológica para reducir de forma significativa tales emisiones. En el influente de la laguna (efluente de la depuradora de Arganda) la concentración de cromo siempre fue superior a la recomendada para aguas de riego de uso continuado; la concentración de boro presentó valores superiores durante el mes de julio, mientras que el hierro y el zinc nunca alcanzaron los umbrales de riesgo (National Academy of Science \& National Academy of Engineering, 1973; WHO, 1984; Ayers y Westcot, 1985; U.S. EPA, 1992). Las concentraciones medias de cromo y boro en el efluente de la laguna no sobrepasaron, sin embargo, los valores de riesgo, si bien, el cromo alcanzó valores ligeramente superiores en momentos puntuales de la mezcla. En próximas campañas se pretende trabajar con tiempos de residencia superiores a 15 días a fin de comprobar si este factor contribuye a incrementar los rendimientos en la eliminación de cromo.

\section{CONCLUSIONES}

- El lagunaje profundo actúa como una trampa de sedimento para los metales pesados. Los procesos de asociación con la materia particulada por adsorción, precipitación, coprecipitación o asimilación por la biota, favorecen su 
retirada de la columna de agua y su acumulación en el sedimento. El diseño y la dinámica natural de la laguna posibilitan la consecución de tales procesos.

- Los rendimientos medios anuales del lagunaje profundo en la eliminación de cromo, hierro y zinc se estimaron próximos al $60 \%$. Durante el proceso completo de regeneración (físico-químico + fangos activos + lagunaje profundo) se obtendrían unos rendimientos totales del $98 \%$ para el cromo, del $92 \%$ para el hierro y el $97 \%$ para el zinc. Juanicó et al. (1999) obtuvieron reducciones del $75 \%$ para el cromo y del $65 \%$ para el zinc un sistema comparable, constituido por dos embalses en serie de $10.4 \mathrm{~m}$ de profundidad, alimentado con agua residual tratada mediante fangos activos.

- Las concentraciones medias de elementos traza en el efluente de la laguna no sobrepasaron los valores de riesgo para la reutilización en riego agrícola.

- No se observaron diferencias significativas entre la mezcla y la estratificación de la laguna en la remoción de elementos traza.

- Los elementos que se detectaron en el sedimento de las trampas fueron los siguientes: cromo, hierro, zinc, níquel, boro, cobre, manganeso, plomo, titanio y cobalto. El cromo fue el elemento que presentó la concentración más elevada en las trampas (12.2 $\pm 0.2 \mathrm{~g} \mathrm{~kg}^{-1}$ MS, en 10 meses de ensayo).

- Según el grado de contaminación del sedimento de la laguna por cromo, zinc, níquel y cobre, éste puede clasificarse como sedimento fuertemente contaminado. Sin embargo, el vaciado de la laguna y la gestión de sus fangos como residuo tóxico sólo sería necesario tras largos periodos de funcionamiento (15-20 años).

\section{BIBLIOGRAFÍA}

ARAUZO, M. 2000. Biotic and abiotic features of a deep wastewater treatment lagoon used for agricultural irrigation. Water Environ. Res., 72(2): 243-247.

ARAUZO, M. 2002. Harmful effects of un-ionised ammonia on the zooplankton community in a deep waste treatment pond. Water Res. (en prensa).

ARAUZO, M., M. F. COLMENAREJO, P. HERNÁIZ, E. MARTÍNEZ \& M. G. GARCÍA. 2000. The role of algae in a deep wastewater self-regeneration pond. Water Res., 34(14): 3666-3674.

ARAUZO, M., M. VALLADOLID \& E. MARTÍNEZ. 2001. Lagunaje profundo: dinámica interna y rendimiento en el proceso de autorregeneración del agua residual. Residuos, 58: 72-77.

APHA, 1998. Standard methods for the examination of water and wastewater. $20^{\text {th }}$ Edition. American Public Health Association, American Water Works Association \& Water Environment Federation. USA.

AYERS, R. S. \& D. W. WESTCOT. 1985. Water quality for agriculture. Food and Agriculture Organization of the United Nations. Rome. $174 \mathrm{pp}$.

BABICH, H. \& G. STOTZKY. 1983. Physicochemical factors of natural reservoirs affect the transformation and exchange of heavy metals toxic to microbes. Envirom. Biogeochem. Ecol. Bull., 35: 316-323.

BAOZHEN, W. 1987. Development of ecological wastewater treatment and utilization systems (EWTUS) in China. Wat. Sci. Technol., 19: 51-63.

BUHR, H. O. \& S. B. MILLER. 1983. A dynamic model of high-rate algal-bacterial wastewater treatment pond. Water Res., 17: 29-37.

CARRE, J., D. BARON, M. LEGEAS, \& J. MAURIN. 1986. Heavy metal, bacterial and viral contaminating of sewage sludge in oxidation ponds. Environ. Technol. Lett., 7(2): 119-127.

DINGES, R. 1982. Natural systems for water pollution control. Van Nostrand Reinhold Co., Nueva York.

DOR, I., I. KALINSKY, J. EREN, \& C. DIMENTMAN. 1987. Deep wastewater reservoirs in Israel; Limnological changes following self-purification. Water Sci. Technol., 19(12): 317-322.

HAMDY, Y. \& L. POST. 1985. Distribution of mercury trace organics and other heavy metals in Detroit River sediments. J. Great Lakes Res., 11(3): 353-365.

INSTITUTO DE INGENIERÍA SANITARIA, 1971. Lagunas de estabilización. Universidad de Buenos Aires. Buenos Aires. 136 pp.

JUANICÓ, M., R. RAVID \& Y. AZOV. 1999. Trace metals. In: Reservoirs for wastewater storage and reuse. Ecology, performance and engineering design. M. Juanicó \& I. Dor, (eds.): 219-232. Springer, Berlin, 394 pp.

KAPLAN, D., A. ABELIOVICH \& S. BEN-YAAKOV. 1987. The fate of heavy metals in wastewater stabilization ponds. Wat. Res., 21(10): 1189-1194.

MARKER, A. F. H., E. A. NUSCH, H. RAI \& B. RIE- 
MANN. 1980. The measurement of photosynthetic pigments in freshwaters and standardization of methods: conclusions and recommendations. Arch. Hydrobiol. Ergebn. Limnol., 14: 91-106.

MIDDLEBROOKS, E. J., C. H. MIDDLEBROOKS, J. H. REYNOLDS, G. Z. WATERS, S. C. REED \& D. B. GEORGE. 1982. Wastewater statabilization lagoon design, performance and upgrading. McMillan Pub. Co., New York.

MORENO, M. D. 1984. Aplicación de estanques profundos a la depuración de aguas residuales urbanas. Tesis Doctoral, Universidad de Murcia.

MORENO, M. D. 1991. Depuración por lagunaje de aguas residuales. Manual de operadores. Dirección General de Medio Ambiente. MOPT. Madrid. 169 pp.

MORENO, M. D., A. SOLER, J. SÁEZ, \& P. ROMERA. 1984a. Study on the hydrodynamic behaviour of a deep waste stabilization pond. Tribune $d u$ Cebedeau, 489-490: 323-328.

MORENO, M. D., A. SOLER, J. MORENO \& J. SÁEZ. 1984b. Thermal simulation of deep stabilization ponds. Tribune du Cebedeau, 490: 367-369.

MORENO, M. D., M. A. MEDINA, J. MORENO, A. SOLER \& J. SÁEZ. 1988. Modeling the performance of deep waste stabilization ponds. Water Resour. Bull., 24(2):377-387.

NATIONAL ACADEMY OF SCIENCE \& NATIONAL ACADEMY OF ENGINEERING. 1973. Water quality criteria 1972. Report of the Committee on water quality criteria. Ecological Research Series. EPA-R373-003. U.S.EPA, Washington, 594 pp.

PALMER, S. A. K., T. J. NUNNO, M. A. BRETON, D. M. SULLIVAN \& N. F. SURPRENANT. 1988. Metal/cyanide containing wastes: Treatment technologies. Pollution Technology Review 158. Noyes Data Corporation, New Yersey, USA. 721 pp.

PARKER, C. D. 1979. Biological mechanisms in lagoons. Prog. Wat. Tech., 11: 71-85.

PARKER, J. G. \& B. J. LYONS. 1979. Factors influencing the treatment of food processing wastes by anaerobic-aerobic lagoon systems. Prog. Wat. Tech., 11: 377-388.

PROSI, F. 1981. Heavy metals in aquatic organisms. In: Metal pollution in the aquatic environments. U. Föstner \& G. T. W. Wittmann (eds.): 271-323. Springer-Verlag, Berlin.

ROVIRA, V. 1993. Contaminación por metales pesados en los sedimentos del río Jarama y su bioasimilación por Tubifícidos (Annelida: Oligochaeta, Tubificidae). Tesis Doctoral, Universidad
Complutense de Madrid. 357 pp.

SMILLE, R. H. \& M. LOUTIT. 1982. Removal of metal from sewage in an oxidation pond system. N. Z. J. Sci., 25(4): 371-376.

SOLER, A., J. SÁEZ, M. LLORENS \& Y. MARTÍNEZ. 1988. Evolución de los parámetros físico-químicos y microbiológicos en la depuración de aguas residuales por lagunaje profundo. Tecnología del Agua, 48: 5258.

STUMM, W. \& J. J. MORGAN. 1981. Aquatic Chemistry. John Wiley \& Sons, New York. 780 pp.

TESSIER, A. \& P. G. CAMPBELL, 1987. Partitioning of trace metals in sediments: relationship with bioavailability. Hydrobiologia, 149. 43-52.

UHLMANN, D. 1979. BOD removal rates of waste stabilization ponds as a function of loading, retention time, temperature and hydraulic flow pattern. Water Res., 13: 193-200.

ULLOA, J. J. 1993. El lagunaje. In: Tratamiento de aguas residuales, basuras y escombros en el ámbito rural. Tragsatec \& Colegio Oficial de Ingenieros Agrónomos (eds.): 69-105. Editorial Agrícola Española, Madrid.

U.S. EPA. 1992. Manual of guidelines for water reuse. U.S. Environmental Protection Agency 625/R92/004. Center for Environmental Research Information. Cincinnati, Ohio, 247, pp.

U.S. EPA. 1998. Toxicological Review of hexavalent chromium. U.S. Environmental Protection Agency 18549-29-9. Washington, DC.

UTERMÖHL, H. 1958. Zur Vervollkomnung der quantitativen Phytoplankton-Meyhodik. Mitt. Interant. Ver. Limnol., 9: 1-38.

VANDER BORGHT, P. \& J. L. VASEL (eds.) 1987. Wastewater treatment in waste stabilization ponds and aerated lagoons. Proceedings of Interuniversity Seminar, Arlon 16-19 Nov. 1987. Fondation Universitaire Luxemburgeoise \& Edictions Cebedoc. 133 pp.

W.H.O. 1984. Health aspects of reuses of treated wastewater for irrigation (background document). WHO. Intercountry Seminar on Wastewater Reuse. Manama, 29 September - 2 October 1984, 51 pp.

WOOD, J. M. 1974. Biological cycles for toxic elements in the environment. Science, 183: 10491052.

WOOD, J. M. 1989. Transport, bioaccumulation and toxicity of elements in microorganisms under environmental stress. Proc. Int. Conf. Heavy Metals in the Environment, Geneva. Vernet (ed.), CEP Consultants Ltd, Edinburgh, UK: 1-12. 
